\title{
Avoided Ferromagnetic Quantum Critical Point: Unusual Short-Range Ordered State in CeFePO
}

\author{
S. Lausberg, ${ }^{1, *}$ J. Spehling, ${ }^{2}$ A. Steppke, ${ }^{1}$ A. Jesche,${ }^{1, \dagger}$ H. Luetkens, ${ }^{3}$ A. Amato, ${ }^{3}$ C. Baines,${ }^{3}$ \\ C. Krellner, ${ }^{1,+}$ M. Brando, ${ }^{1}$ C. Geibel, ${ }^{1}$ H.-H. Klauss, ${ }^{2}$ and F. Steglich ${ }^{1}$ \\ ${ }^{1}$ Max-Planck-Institute for Chemical Physics of Solids, D-01187 Dresden, Germany \\ ${ }^{2}$ Institute for Solid State Physics, TU Dresden, D-01069 Dresden, Germany \\ ${ }^{3}$ Laboratory for Muon-Spin Spectroscopy, Paul-Scherrer-Institute, CH-5232 Villigen, Switzerland
}

(Received 5 June 2012; published 20 November 2012)

\begin{abstract}
Cerium $4 f$ electronic spin dynamics in single crystals of the heavy-fermion system $\mathrm{CeFePO}$ is studied by means of ac susceptibility, specific heat, and muon-spin relaxation $(\mu \mathrm{SR})$. Short-range static magnetism occurs below the freezing temperature $T_{g} \approx 0.7 \mathrm{~K}$, which prevents the system from accessing a putative ferromagnetic quantum critical point. In the $\mu \mathrm{SR}$, the sample-averaged muon asymmetry function is dominated by strongly inhomogeneous spin fluctuations below $10 \mathrm{~K}$ and exhibits a characteristic time-field scaling relation expected from glassy spin dynamics, strongly evidencing cooperative and critical spin fluctuations. The overall behavior can be ascribed neither to canonical spin glasses nor other disorder-driven mechanisms.
\end{abstract}

DOI: 10.1103/PhysRevLett.109.216402

PACS numbers: 71.27.+a, 64.70.Tg, 75.50.Lk, 76.75.+i

A long-standing question in the field of quantum criticality is whether a ferromagnetic (FM) quantum critical point (QCP) generally exists and, if not, which are the possible ground states of matter that replace it. Quantum critical points occur when a material is continuously tuned with an external parameter (pressure, magnetic field, etc.) between competing ground states at zero temperature $[1,2]$. A FM QCP then exists when it is possible to shift the Curie transition temperature $T_{C}$ of a ferromagnet continuously to zero where a second order quantum phase transition takes place. Quantum phase transitions occur at zero entropy and are driven by quantum rather than thermal fluctuations. These fluctuations diverge at the QCP modifying the excitation spectrum of a metal and leading to a fundamental instability of Landau's Fermi liquid (FL) [3]. Typical signatures of such a behavior are observed in magnetic, thermal, and transport properties and are referred to as non-Fermi-liquid (NFL) phenomena [4].

Although there is clear evidence for the existence of antiferromagnetic (AFM) QCPs, the FM QCP case is controversial. In recent years, substantial experimental and theoretical efforts were made to further investigate this problem. However, a wide range of possibilities exists. On theoretical grounds, a 3D FM QCP is believed to be inherently unstable, either towards a first order phase transition or towards an inhomogeneous magnetic phase (modulated or textured structures) [5-7]. Similar results have been obtained in 2D [5,8,9]. Several clean (stoichiometric) magnetic transition-metal compounds, like $\mathrm{MnSi}$ [10] or $\mathrm{ZrZn}_{2}$ [11], show NFL behavior close to a FM instability, but the transition changes into a first order one. In other systems the existence of a FM QCP has been proposed, most notably in $\mathrm{Nb}_{1-y} \mathrm{Fe}_{2+y}$ [12], $\mathrm{Zr}_{1-x} \mathrm{Nb}_{x} \mathrm{Zn}_{2}$ [13], or $\mathrm{SrCo}_{2}\left(\mathrm{Ge}_{1-x} \mathrm{P}_{x}\right)_{2}$ [14] where the FM QCP is attained by chemical substitution. However, in these cases the influence of disorder remains ambiguous.

More appropriate candidates for the study of FM QCPs are $\mathrm{U}, \mathrm{Yb}$, or $\mathrm{Ce}$ based $f$-electron metals [2,4], since in these materials the NFL signatures are much more pronounced due to their heavy-fermion character. However, while there is quite a number of $U$ based systems showing either a first order FM transition $\left(\mathrm{UGe}_{2}, \mathrm{UCoAl}, \mathrm{UCoGe}\right)$ [15] or indications for a FM QCP $\left(\mathrm{UCu}_{5-x} \mathrm{Pd}_{x}\right.$ [16], $\mathrm{URh}_{1-x} \mathrm{Ru}_{x} \mathrm{Ge}$, [17]), the number of $\mathrm{Yb}$ based systems close to a FM QCP is very limited $\left(\mathrm{YbNi}_{4} \mathrm{P}_{2}\right.$ [18], $\mathrm{YbCu}_{2} \mathrm{Si}_{2}$ [19]). Several systems, like $\mathrm{CeRu}_{2} \mathrm{Ge}_{2}$ [20] or CeRuPO [21] where the FM transition temperature is suppressed to $T=0$ by hydrostatic pressure, exhibit a change into AFM order before reaching the QCP. There are Ce based alloys $\left(\mathrm{CePd}_{1-x} \mathrm{Rh}_{x}\right.$ [22] ) and also $d$-electron metals $\left(\mathrm{Ni}_{1-x} \mathrm{~V}_{x}\right.$ [23]) where it seems that local disorder-driven mechanisms such as Kondo disorder or the quantum Griffiths phase (QGP) scenario are responsible for the NFL properties [24-26]. Broad and strongly $T$-dependent NMR and muon-spin relaxation $(\mu \mathrm{SR})$ linewidths are indicative for such disorder-driven mechanisms. As a consequence, spin-glass-like behavior is often found, e.g., in $\mathrm{CePd}_{1-x} \mathrm{Rh}_{x}$, and power-law corrections to the thermodynamic and transport properties as well as in the local spin dynamics are expected in a broad region across the putative QCP. The global phase transition then becomes smeared $[22,27,28]$.

In this context, the layered Kondo-lattice system $\mathrm{CeFePO}$ is a unique candidate for studying FM QCPs, since it is a clean nonmagnetic (nonsuperconducting) heavy-fermion metal located very close to a FM instability with strong FM fluctuations [29,30]. CeFePO is a homologue of the quaternary iron pnictides. It evolves from a 
long-range ordered FM ground state, when a small amount of arsenic is substituted for phosphorus [31-33]. Less As concentration leads to a continuous decrease of $T_{C}$ culminating into a putative FM QCP. The isovalent As substitution not only introduces a volume effect (shortening mostly the $c$ axis) but also increases locally the hybridization strength between the trivalent $\mathrm{Ce} 4 f$ and the Fe $3 d$ conduction electrons leading to an enhancement of the Kondo temperature, which is approximately $10 \mathrm{~K}$ for $\mathrm{CeFePO}$ [34]. Below this temperature, the susceptibility and the Knight shift become field dependent and the NMR line width broadens. This identifies the onset of short-range FM correlations essentially within the basal plane, evidencing a strong anisotropy [29], which could also be confirmed by recent NMR measurements on oriented powder [35]. The ground state of $\mathrm{CeFePO}$ was found to be a paramagnetic heavy FL [29].

In this Letter, we present a comprehensive study of the ac susceptibility $\left(\chi^{\prime}\right)$, specific heat $(C)$ and $\mu$ SR measurements on recently grown high-quality single crystals of $\mathrm{CeFePO}$. We find evidence of strongly inhomogeneous spin fluctuations starting below $10 \mathrm{~K}$ and of a spin-glasslike freezing at $T_{g} \approx 0.7 \mathrm{~K}$ which prevents the system from accessing the putative FM QCP. The observed timefield scaling of the muon asymmetry points to a cooperative mechanism and to the presence of critical spin fluctuations. The overall behavior can not be ascribed to either canonical spin glasses or to other disorder-driven mechanisms. The physics of $\mathrm{CeFePO}$ is different from other candidate systems where the putative FM QCP is avoided by a first order phase transition or a transition into an AFM state.

The samples were synthesized by means of a two-step Sn flux method. The small crystals were powdered and pressed into pellets which are referred to as polycrystalline (PC) sample in the subsequent discussion. The large crystals were oriented and glued together with silver paint to form a larger "single crystal" (SC). X-ray powder diffraction confirms the $\mathrm{ZrCuSiAs}$ structure type [31]. The synthesis conditions were different from the samples investigated previously $[29,31]$. However, the samples PC and SC as well as the sample of Ref. [29] are indistinguishable within the resolution of energy dispersive x-ray measurements which confirm the stoichiometric ratio 1:1:1:1. Low temperature $\chi^{\prime}(T, B), C(T, B)$, and $\mu \operatorname{SR}(T, B)$ were measured in dilution refrigerators. A commercial SQUID vibrating sample magnetometer (Quantum Design) was used to measure $\chi^{\prime}$ above $1.8 \mathrm{~K}$. The $\mu$ SR experiments were performed on the $\pi \mathrm{M} 3$ beam line at the Swiss Muon Source at the Paul-Scherrer-Institut, Switzerland. They were executed in zero magnetic field and in applied magnetic fields up to $0.75 \mathrm{~T}$ parallel to the initial muon-spin polarization (LF $\mu \mathrm{SR}$ ).

The first evidence of spin freezing is seen in the $T$ dependence of $\chi^{\prime}(T)$ for the PC sample [Fig. 1(a)]. At $B=0$ a distinct peak is found at $T_{g}=0.67(1) \mathrm{K}$
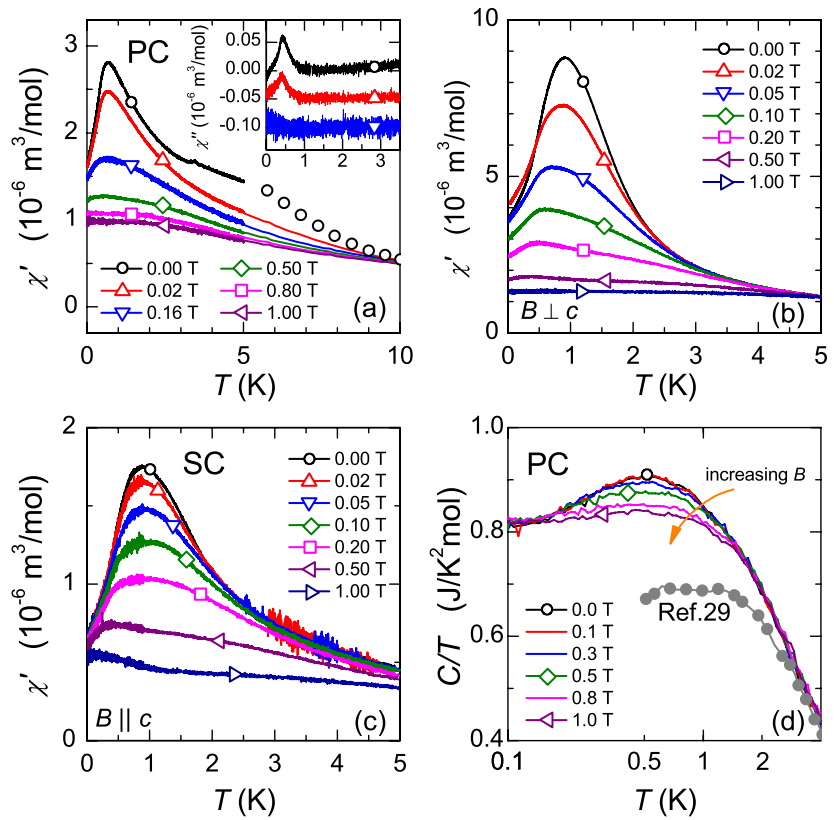

FIG. 1 (color online). (a) ac susceptibility $\chi^{\prime}(T)$ of the PC sample with its imaginary part $\chi^{\prime \prime}(T)$ (inset). (b) $\chi^{\prime}(T)$ vs $T$ for the SC sample with $B \perp c$. (c) $\chi^{\prime}(T)$ vs $T$ for the SC sample with $B \| c$. (d) Specific heat of the PC sample plotted as $C / T$ vs $T$.

where the susceptibility reaches values as high as $2.8 \times 10^{-6} \mathrm{~m}^{3} / \mathrm{mol}$. The $\chi^{\prime}(T)$ value at $2 \mathrm{~K}$ is three times larger than the one measured in the sample of Ref. [29]. With increasing field, its amplitude decreases and $T_{g}$ shifts slightly and, above $0.8 \mathrm{~T}, \chi^{\prime}(T)$ flattens. The small hump between 3 and $9 \mathrm{~K}$ is only seen at $B=0$ in the PC sample. It is most likely due to an impurity phase, as it is completely suppressed in a small field of $0.02 \mathrm{~T}$ while $T_{g}$ does not change. Moreover, a comparable feature is absent in the SC case. Dissipative effects are corroborated by a peak in $\chi^{\prime \prime}(T)$ at about $0.45 \mathrm{~K}$ [inset of Fig. 1(a)], which is a significantly lower temperature than that of the maximum in $\chi^{\prime}(T)$. The same effects and $B$ dependence are observed in the SC sample [Fig. 1(b)], but at a higher $T_{g}=$ $0.92(2) \mathrm{K}$. This could be due to a very tiny difference in stoichiometry, since both the PC and SC samples were taken from small and large crystals, respectively, of the same batch which likely form at different times during the growth. The susceptibility is very anisotropic $\left(\chi_{\perp c} / \chi_{\|_{c}} \approx 5\right)$, and with $B \perp c$ it reaches a high peak value of $9 \times 10^{-6} \mathrm{~m}^{3} / \mathrm{mol}$ [Figs. 1(b) and 1(c)] which is much larger than in the PC case. This observation confirms the presence of anisotropic FM spin fluctuations, which are much stronger along the basal planes. To check the bulk nature of the freezing, we have measured the specific heat of the PC sample [Fig. 1(d)]. A broad maximum emerges at about $0.55 \mathrm{~K}$ in a $C / T$ vs $T$ plot. While at $0.1 \mathrm{~T}$ the maximum is unchanged, larger fields suppress it. The entropy difference between the zerofield and the high-field curves is small, about $1 \%$ of $R \ln (2)$, 


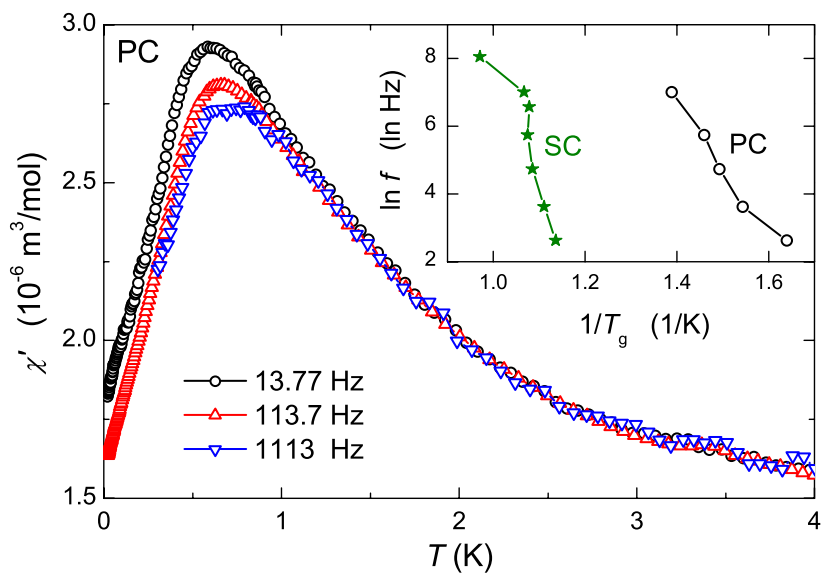

FIG. 2 (color online). Frequency dependence of $\chi^{\prime}(T)$ for the PC sample. Inset: Arrhenius plot for the PC sample and for the SC sample with $B \perp c$.

which is due to the Kondo screening of the Ce moments. The data of the polycrystalline sample investigated in Ref. [29] [solid circles in Fig. 1(d)] resemble the $C / T$ behavior at high temperatures, while below $3 \mathrm{~K}, C / T$ remains constant without any indication of freezing.

We have performed frequency $(f)$ dependent measurements at $B=0$ on the PC and SC samples to investigate the spin dynamics (Fig. 2). Similar to spin glasses, the maximum in $\chi^{\prime}(T)$ shifts to higher temperatures as the excitation frequency is increased, while its amplitude decreases. $T_{g}$ and $f$ are shown in an Arrhenius plot in the inset of Fig. 2, from which the frequency shift $\delta$ [36] can be evaluated. For the PC and the SC sample we obtain $\delta_{\mathrm{PC}}=0.085(11)$ and $\delta_{\mathrm{SC}}=0.065(16)$, respectively. These values are larger than those found for canonical spin glasses $(\delta \approx$ $0.005-0.06)$, yet they are below typical values of superparamagnets $(\delta \approx 0.3)$ [36]. A qualitatively similar behavior was found for $\mathrm{CePd}_{1-x} \mathrm{Rh}_{x}$, where $\delta$ increases when approaching the critical point at $x=0.87$ [22]. It is interesting to note that in $\mathrm{CeFePO}$ the sample with lower $T_{g}$ exhibits larger $\delta$, too. This suggests that the QGP scenario might be also applicable in CeFePO. On the other hand, the magnetic anisotropy and the lack of evidence for FM cluster formation in zero-field-cooled and field-cooled magnetization measurements (not shown) rule out such a mechanism in $\mathrm{CeFePO}$. Moreover, we could not fit our data with $\chi \propto C / T \propto T^{\lambda-1}$ with $0 \leq \lambda \leq 1$ in any reasonable $T$-range [25].

To study the microscopic nature of the low- $T$ magnetism in $\mathrm{CeFePO}, \mu \mathrm{SR}$ measurements in zero and small longitudinal fields were performed on the PC sample. $\mu \mathrm{SR}$ in small fields is dominated by thermally excited Ce $4 f$ electronic spin fluctuations that couple to the implanted muons. Figure 3(a) displays the temperature evolution of the normalized muon-spin asymmetry function $G(t, B)$ at a constant field of $B=0.01 \mathrm{~T}$ which is sufficient to quench the weak static relaxation due to nuclear dipole fields at the

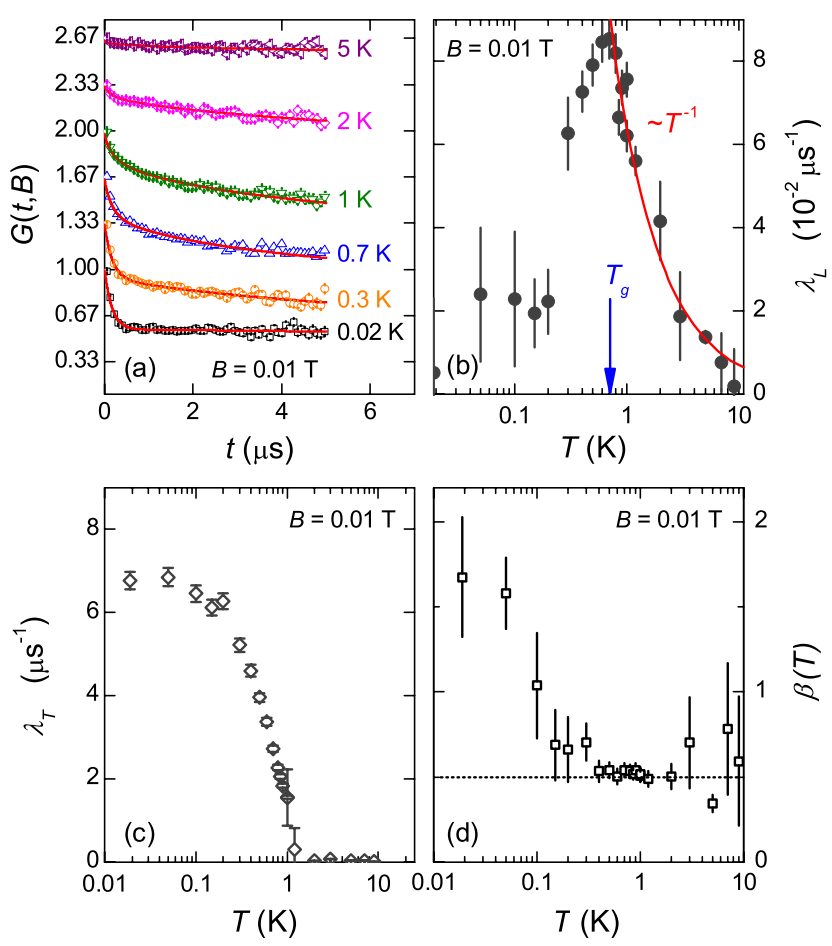

FIG. 3 (color online). (a) Normalized muon-spin asymmetry function $G(t, B)$ at an applied field $B=0.01 \mathrm{~T}$ for representative temperatures above and below $T_{g}$. Solid lines are fits according to Eq. (1). For clarity, the different curves are shifted subsequently by 0.33 . (b) $T$ dependence of the dynamic $\mu$ SR rate $\lambda_{L}$. The arrow marks the magnetic transition from dynamic to static magnetism at $T_{g}=0.70(3) \mathrm{K}$. (c) $T$ dependence of the static $\mu \mathrm{SR}$ rate $\lambda_{T}$. (d) $T$ dependence of the exponent $\beta$ in Eq. (1). The dotted line denotes the value $\beta=0.5$.

muon sites, leaving only the dynamic and static contributions due to the electronic $\mathrm{Ce} 4 f$ magnetic moments. The quantitative analysis takes into account both static and dynamical fields: The static relaxation dominates $G(t, B)$ at short times $t$, while at long $t$ the relaxation rate probes only the dynamic spin fluctuations, i.e., the Fourier transform of the dynamic spin-spin autocorrelation function $q(t)=\left\langle\mathbf{S}_{i}(t) \cdot \mathbf{S}_{i}(0)\right\rangle$. To account for both contributions we use the following fitting function

$$
G(t, B)=G_{1} \exp \left[-\left(\lambda_{T} t\right)\right]+G_{2} \exp \left[-\left(\lambda_{L} t\right)^{\beta}\right],
$$

with static (transversal) and dynamic (longitudinal) relaxation rates $\lambda_{T}$ and $\lambda_{L}$, respectively. The fits provide a very good description of the experimental data [solid lines in Fig. 3(a)]. At low $T$ the spectra show nearly no muon-spin relaxation at long times, i.e., very small relaxation rates $\lambda_{L}$. Upon increasing $T, \lambda_{L}$ increases and reaches a maximum at $T_{g}=0.70(3) \mathrm{K}$ [Fig. 3(b)] in agreement with $T_{g}$ found in $\chi^{\prime}(T)$. Subsequently, it decays following a $T^{-1}$ behavior up to $10 \mathrm{~K}$ after which no dynamic relaxation is observed in accordance with NMR and susceptibility experiments [29]. On the contrary, the static component $\lambda_{T}$ increases 
steeply below $T_{g}$ up to a value of $7 \mu \mathrm{s}^{-1}$ [Fig. 3(c)]. Its behavior resembles that of the magnetic order parameter when entering a magnetically ordered phase. The $\beta$ value of about 0.5 for $T \geq T_{g}$ [Fig. 3(d)] indicates a broad inhomogeneous distribution of fluctuating dynamical local fields (or relaxation rates) [37]. In the ordered phase $\beta$ increases, reaching a value of about 1.7 at $T=0.02 \mathrm{~K}$, indicating that the spin fluctuations become static. Figure 4(a) displays $G(t, B)$ at $0.02 \mathrm{~K}$. At $B=0$, the absence of a spontaneous muon-spin precession frequency indicates short-range magnetic order. Such a strongly damped $\mu$ SR signal allows for an estimation of the magnetic coherence length $\xi<10 a$, where $a$ is the lattice constant [38]. The observation of a $2 / 3$ and $1 / 3$ signal fraction below $T_{g}$ proves that $100 \%$ of the sample volume shows static magnetic order [39]. The muon-spin relaxation is completely suppressed at $B=0.75 \mathrm{~T}$ demonstrating that the internal field distribution is static in nature at $T=0.02 \mathrm{~K}$. Increasing $T$, a dynamic contribution to the muon-spin relaxation develops [Fig. 4(b)]. Glassy spin dynamics generally result in long-time correlations with distinct signatures when $T_{g}$ is approached from high $T$ [40]. Theoretically, $q(t)$ is predicted to exhibit powerlaw $q(t)=c t^{-\alpha}$ or stretched exponential $q(t)=$ $c \exp \left[-(\Lambda t)^{K}\right]$ behavior at $T>T_{g}$, that in both cases can lead to a characteristic time-field scaling $G(t, B)=$ $G\left(t / B^{\gamma}\right)$ after Fourier transforming $q(t)$, where $\gamma<1$ and $\gamma>1$ for power-law and stretched exponential correlations, respectively [40]. If this equation is obeyed a plot of $G(t, B)$ vs $t / B^{\gamma}$ at $T>T_{g}$ will be universal. Figure 4(b) displays $G(t, B)$ at $T=0.9 \mathrm{~K}$, which is slightly above $T_{g}$, both in zero field and in magnetic longitudinal field between 0.01 and $0.75 \mathrm{~T}$. The observed $B$ dependence corresponds to a measurement of the Fourier transform of $q(t)$ over the frequency range $\gamma_{\mu} B / 2 \pi \approx 1.4-100 \mathrm{MHz}$, where $\gamma_{\mu}=2 \pi \times 135.53 \mathrm{MHz} / \mathrm{T}$ is the muon gyromagnetic

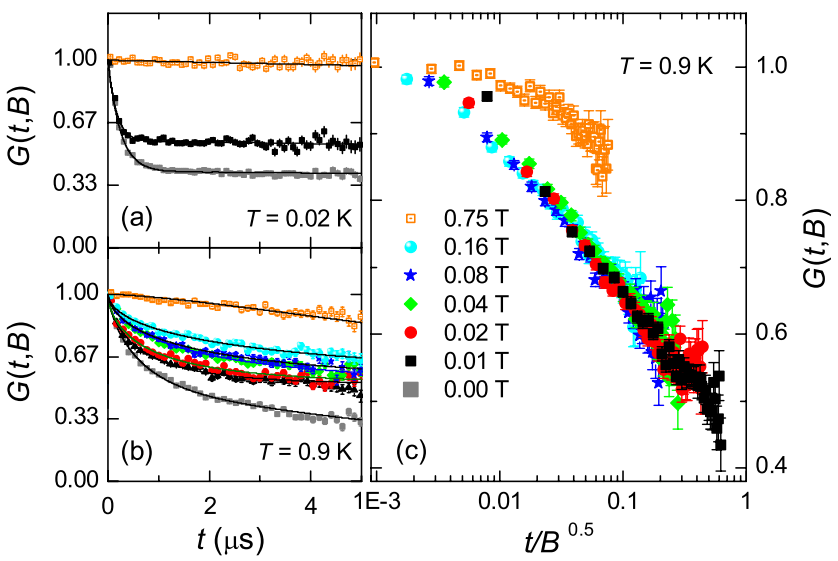

FIG. 4 (color online). (a) Field dependence of the muon-spin asymmetry function $G(t, B)$ in $\mathrm{CeFePO}$ at $T=0.02 \mathrm{~K}$ and (b) $T=0.9 \mathrm{~K}$. (c) The same data as in (b), plotted as a function of the scaling variable $t / B^{0.5}$. ratio. As shown, the relaxation slows with increasing $B$. For low enough $B$, the $B$ dependence is expected to be due to the change of $\gamma_{\mu} B$ rather than an effect of field on $q(t)$ [37]. A breakdown of time-field scaling is expected for high fields where $q(t)$ is directly affected by the applied fields. Figure 4(c) shows the same muon-spin asymmetry data as a function of $t / B^{\gamma}$. For $\gamma=0.5(1)$ the data scale well over nearly 2.5 orders of magnitude in $t / B^{\gamma}$ for all applied fields except for $0.75 \mathrm{~T}$, as expected for fields with $\mu_{B} B \geq k_{B} T$, which should affect $q(t)$. The obtained scaling exponent $\gamma=0.5(1)<1$ implies that, within the $\mu$ SR frequency range, $q(t)$ is well approximated by a power law, suggesting cooperative and critical spin fluctuations rather than a distribution of local fluctuation rates [37]. This is in contrast to $\beta=0.5$ which indicates a broad inhomogeneous distribution of local fluctuation rates. The cooperative behavior is supported by the fact that $\mathrm{CeFePO}$ is a stoichiometric system and the narrow NMR linewidth proves that it is locally not disordered [29]. Short-range correlations set in below $10 \mathrm{~K}$, broadening the linewidth, in agreement with the $\mu \mathrm{SR}$ results. The value of $\gamma$ seems to be weakly $T$ dependent (it is 0.4 at about $2 \mathrm{~K}$, not shown) which suggests slow quantum rather than thermal fluctuations. From the $B$ dependence of $\lambda_{L}$, the spin autocorrelation time $\tau_{c}$ can be estimated using $\lambda_{L}(B)=\left(2 \gamma_{\mu}^{2}\left\langle B_{\mathrm{loc}}^{2}\right\rangle \tau_{c}\right) /\left[1+\left(\gamma_{\mu}^{2} B^{2} \tau_{c}^{2}\right)^{p}\right]$ [41]. Here, $B_{\mathrm{loc}}(t)$ describes the time-varying local magnetic field at the muon site due to fluctuations of neighboring Ce $4 f$ moments, with a local time averaged second moment $\gamma_{\mu}\left\langle B_{\text {loc }}^{2}\right\rangle$. For $\hbar \omega \gg k_{B} T$, the fluctuationdissipation theorem relates $\tau_{c}$ to the imaginary component of the local $q$-independent $f$-electron dynamic susceptibility, i.e., $\tau_{c}(B) \approx\left(k_{B} T / \mu_{B}^{2}\right)\left[\chi^{\prime \prime}(\omega) / \omega\right]$ [42]. A fit to the data (not shown) yields $\tau_{c} \approx 1.8 \times 10^{-8}$ s and $p=0.67$. The value for $\tau_{c}$ indicates very slow or glass-type spin dynamics.

In conclusion, we have shown that single crystals of CeFePO, located close to a FM instability, show spinglass-like freezing. It is evidenced by the frequencydependent peak in $\chi^{\prime}(T)$ at $T_{g}$, the broad maximum in $C(T) / T$ as well as clear signatures in $G(t, B)$, which unambiguously show the transition from dynamic to short-range static magnetism. The frequency shift of $T_{g}$ suggests values slightly larger than for canonical spin glasses, indicating the presence of large fluctuating regions above $T_{g}$, and the time-field scaling strongly suggests cooperative behavior. Our results imply that the putative FM QCP is avoided in a new manner: We do not observe a first order phase transition or AFM order, but rather a transition into a shortrange ordered state. Moreover, the magnetic anisotropy, the lack of evidence for FM clusters and the time-field scaling rule out a disorder-driven scenario (e.g., the QGP) as the mechanism underlying the spin dynamics in CeFePO. We might have in CeFePO a combination of both scenarios: The close proximity of $\mathrm{CeFePO}$ to a FM instability and its magnetic anisotropy seem to drive the system 
to develop short-range magnetic correlations which might have their origin in the mechanism described in Refs. [5-7]. Below $T_{g}$, magnetic short-range order then forms with a certain texture (e.g., homogeneous cobbled magnetically ordered regions of different sizes) which would explain the spatially distributed $\mu$ SR rates.

We are indebted to G. J. Conduit, A.G. Green, D.E. MacLaughlin, and T. Vojta for useful discussions, and we acknowledge the PSI accelerator crew. This research project has been supported by the European Commission under the 7th Framework Programme through the "Research Infrastructures" action of the "Capacities" Programme, NMI3-II Grant No. 283883. Part of this work has been supported by the DFG Research Unit 960 "Quantum Phase Transitions" and the SPP 1458 priority program.

Note added in proof.-Recently, we became aware of a study of the related alloy $\mathrm{Ce}\left(\mathrm{Ru}_{1-x} \mathrm{Fe}_{x}\right) \mathrm{PO}$ suggesting a FM QCP in this system near $x=0.86$ Ref. [43].

*lausberg@cpfs.mpg.de

†Present address: Ames Laboratory, U.S. DOE and Department of Physics and Astronomy, Iowa State University, Ames, IA 50011, USA.

Present address: Institute of Physics, Goethe University Frankfurt, D-60438 Frankfurt am Main, Germany.

[1] S. Sachdev, Quantum Phase Transitions (Cambridge University Press, Cambridge, England, 1999).

[2] H. v. Löhneysen, A. Rosch, M. Vojta, and P. Wölfle, Rev. Mod. Phys. 79, 1015 (2007).

[3] G. Baym and C. Pethick, Landau Fermi-Liquid Theory (Wiley, New York, 2004).

[4] G. R. Stewart, Rev. Mod. Phys. 73, 797 (2001).

[5] D. Belitz, T. R. Kirkpatrick, and T. Vojta, Phys. Rev. Lett. 82, 4707 (1999); T. R. Kirkpatrick and D. Belitz, Phys. Rev. B 85, 134451 (2012).

[6] A. V. Chubukov, C. Pépin, and J. Rech, Phys. Rev. Lett. 92, 147003 (2004).

[7] G. J. Conduit, A. G. Green, and B. D. Simons, Phys. Rev. Lett. 103, 207201 (2009).

[8] D. L. Maslov, A. V. Chubukov, and R. Saha, Phys. Rev. B 74, 220402 (2006).

[9] G. J. Conduit, Phys. Rev. A 82, 043604 (2010).

[10] C. Pfleiderer, P. Böni, T. Keller, U.K. Rößler, and A. Rosch, Science 316, 1871 (2007).

[11] M. Uhlarz, C. Pfleiderer, and S. M. Hayden, Phys. Rev. Lett. 93, 256404 (2004).

[12] M. Brando, W. J. Duncan, D. Moroni-Klementowicz, C. Albrecht, D. Grüner, R. Ballou, and F. M. Grosche, Phys. Rev. Lett. 101, 026401 (2008).

[13] D. A. Sokolov, M. C. Aronson, W. Gannon, and Z. Fisk, Phys. Rev. Lett. 96, 116404 (2006).

[14] S. Jia, P. Jiramongkolchai, M. R. Suchomel, B. H. Toby, J. G. Checkelsky, N. P. Ong, and R. J. Cava, Nat. Phys. 7, 207 (2011).
[15] D. Aoki and J. Flouquet, J. Phys. Soc. Jpn. 81, 011003 (2012).

[16] O. O. Bernal, D. E. MacLaughlin, H. G. Lukefahr, and B. Andraka, Phys. Rev. Lett. 75, 2023 (1995).

[17] N. T. Huy, A. Gasparini, J.C.P. Klaasse, A. de Visser, S. Sakarya, and N.H. van Dijk, Phys. Rev. B 75, 212405 (2007).

[18] C. Krellner, S. Lausberg, A. Steppke, M. Brando, L. Pedrero, H. Pfau, S. Tencé, H. Rosner, F. Steglich, and C. Geibel, New J. Phys. 13, 103014 (2011).

[19] A. Fernandez-Pañella, D. Braithwaite, B. Salce, G. Lapertot, and J. Flouquet, Phys. Rev. B 84, 134416 (2011).

[20] S. Süllow, M. C. Aronson, B. D. Rainford, and P. Haen, Phys. Rev. Lett. 82, 2963 (1999).

[21] M. E. Macovei, Ph.D. thesis, University of Dresden, 2010.

[22] T. Westerkamp, M. Deppe, R. Küchler, M. Brando, C. Geibel, P. Gegenwart, A.P. Pikul, and F. Steglich, Phys. Rev. Lett. 102, 206404 (2009).

[23] S. Ubaid-Kassis, T. Vojta, and A. Schroeder, Phys. Rev. Lett. 104, 066402 (2010).

[24] E. Miranda, V. Dobrosavljević, and G. Kotliar, Phys. Rev. Lett. 78, 290 (1997).

[25] A. H. Castro Neto, G. Castilla, and B. A. Jones, Phys. Rev. Lett. 81, 3531 (1998).

[26] T. Vojta, J. Phys. A 39, R143 (2006).

[27] T. Vojta, Phys. Rev. Lett. 90, 107202 (2003).

[28] J. A. Hoyos and T. Vojta, Phys. Rev. Lett. 100, 240601 (2008).

[29] E. M. Brüning, C. Krellner, M. Baenitz, A. Jesche, F. Steglich, and C. Geibel, Phys. Rev. Lett. 101, 117206 (2008).

[30] D. A. Zocco et al., Phys. Rev. B 83, 094528 (2011).

[31] A. Jesche, Ph.D. thesis, University of Dresden, 2011.

[32] A. Jesche et al., Phys. Rev. B 86, 020501 (2012).

[33] Y. Luo, Y. Li, S. Jiang, J. Dai, G. Cao, and Z.-a. Xu, Phys. Rev. B 81, 134422 (2010).

[34] M. G. Holder et al., Phys. Rev. Lett. 104, 096402 (2010).

[35] S. Kitagawa, H. Ikeda, Y. Nakai, T. Hattori, K. Ishida, Y. Kamihara, M. Hirano, and H. Hosono, Phys. Rev. Lett. 107, 277002 (2011).

[36] J. A. Mydosh, Spin Glasses: An Experimental Introduction (Taylor \& Francis, London, 1993).

[37] D. E. MacLaughlin, R. H. Heffner, O. O. Bernal, K. Ishida, J.E. Sonier, G. J. Nieuwenhuys, M. B. Maple, and G. R. Stewart, J. Phys. Condens. Matter 16, S4479 (2004).

[38] A. Yaouanc and P. Dalmas de Réotier, Muon Spin Rotation, Relaxation, and Resonance (Oxford University, New York, 2011).

[39] Please note, a constant $T$-independent background signal due to muons that stopped in a $\mathrm{Ag}$ sample holder (signal fraction $=15 \%$ ) was taken into account.

[40] A. Keren, P. Mendels, I. A. Campbell, and J. Lord, Phys. Rev. Lett. 77, 1386 (1996).

[41] D. T. Adroja, A. D. Hillier, J.-G. Park, W. Kockelmann, K. A. McEwen, B. D. Rainford, K.-H. Jang, C. Geibel, and T. Takabatake, Phys. Rev. B 78, 014412 (2008).

[42] J. S. Toll, Phys. Rev. 104, 1760 (1956).

[43] S. Kitagawa, K. Ishida, T. Nakamura, M. Matoba, and Y. Kamihara, arXiv:1210.4604v1. 